\title{
Molecular Simulations of the Synthesis of Periodic Mesoporous Silica Phases at High Surfactant Concentrations
}

\author{
Szu-Chia Chien, ${ }^{\dagger}$ Germán Pérez-Sánchez, ${ }^{\ddagger}{ }^{\ddagger}$ José R. B. Gomes, ${ }^{\ddagger}$ (๑) M. Natália D. S. Cordeiro, ${ }^{\S}$ \\ Miguel Jorge, ${ }^{*}, \|^{\oplus}$ Scott M. Auerbach, ${ }^{*},+\perp$ and Peter A. Monson ${ }^{*} \dagger$
}

${ }^{\dagger}$ Department of Chemical Engineering and ${ }^{\perp}$ Department of Chemistry, University of Massachusetts, 686 North Pleasant Street, Amherst, Massachusetts 01003-9303, United States

*CICECO-Aveiro Institute of Materials, Department of Chemistry, University of Aveiro, Campus Universitário de Santiago, 3810-193 Aveiro, Portugal

${ }^{\S}$ LAQV@REQUIMTE, Department of Chemistry and Biochemistry, Faculty of Sciences, University of Porto, Rua Campo Alegre 687, 4169-007 Porto, Portugal

"Department of Chemical and Process Engineering, University of Strathclyde, 75 Montrose Street, Glasgow G1 1XJ, United Kingdom

Supporting Information

ABSTRACT: Molecular dynamics simulations of a coarse-grained model are used to study the formation mechanism of periodic mesoporous silica over a wide range of cationic surfactant concentrations. This follows up on an earlier study of systems with low surfactant concentrations. We started by studying the phase diagram of the surfactant-water system and found that our model shows good qualitative agreement with experiments with respect to the surfactant concentrations where various phases appear. We then considered the impact of silicate species upon the morphologies formed. We have found that even in concentrated surfactant systems - in the concentration range where pure surfactant solutions yield a liquid crystal phase-the liquid-crystal templating mechanism is not viable because the preformed liquid crystal collapses as silica monomers are added into the solution. Upon the addition of silica dimers, a new phaseseparated hexagonal array is formed. The preformed liquid crystals were found to be unstable in the presence of monomeric silicates. In addition, the silica dimer is found to be essential for mesoscale ordering at both low and high surfactant concentrations. Our results support the view that a cooperative interaction of anionic silica oligomers and cationic surfactants determines the mesostructure formation in the M41S family of materials.
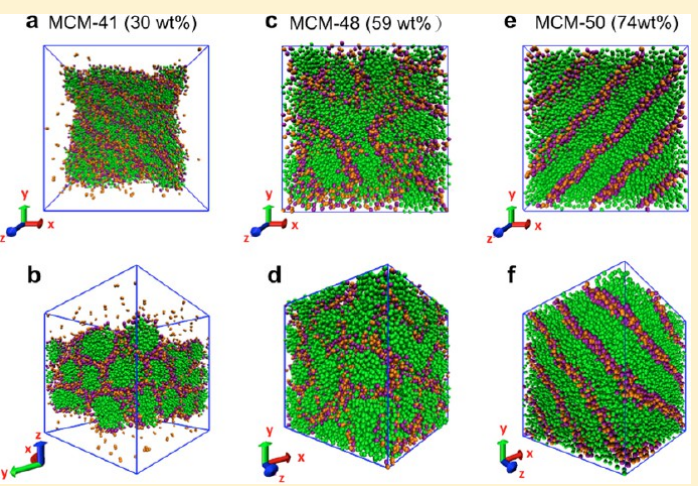

\section{INTRODUCTION}

Periodic mesoporous silicas (PMSs) are widely used in many applications such as catalysis, membrane separation, and drug vehicles. ${ }^{1}$ These materials are usually fabricated through the self-assembly of surfactants (usually cetyltrimethylammonium bromide, or CTAB) and silicate precursors in solution. Varying the synthetic conditions allows us to control important properties such as pore size, shape, and wall thickness. In order to be able to tailor PMS materials for particular applications, significant effort has been dedicated to investigating various aspects of MCM-41, perhaps the most popular material of its class, including its pore structure, pore properties, structural modification, and formation mechanisms. ${ }^{2-6}$ Two formation mechanisms of MCM-41 have been postulated from experimental observations: the cooperative templating mechanism $(\mathrm{CTM})^{7,8}$ and the liquid-crystal templating (LCT) mechanism. ${ }^{9}$ The CTM suggests that silicates play a crucial role during the formation of the MCM-41 mesostructure-the interaction of anionic silicates and cationic surfactants drives the formation of the hexagonal array from an initial solution of individual micelles. On the other hand, the LCT mechanism suggests that the structure of preformed surfactant liquid crystals determines the MCM-41 structure, while silicates simply condense around these preformed mesophases. ${ }^{9,10}$ Even though a huge number of experiments have been carried out to explore the MCM-41 formation, elucidation of the selfassembly of MCM-41, a very complex process that includes the chemical and physical interactions among different species in solution, remains challenging. The comprehensive understanding of the formation mechanism is still incomplete.

Molecular simulations have been used to study the selfassembly process of PMS formation via exploring the organic/ inorganic species interactions. Larson and co-workers developed a simple chainlike surfactant model together with lattice Monte Carlo simulations to study the formation of surfactant liquid crystalline phases in surfactant solutions. ${ }^{11}$ This

Received: September 18, 2016

Revised: January 30, 2017

Published: February 7, 2017 
surfactant model was later extended by Siperstein and Gubbins ${ }^{12,13}$ to study organic-inorganic-solvent three-component systems, where the inorganic component was meant to represent silicates. Phase separation was found between a solvent-rich phase and a concentrated phase rich in silicates and surfactant. In their simulations, different liquid crystals, such as hexagonal and lamellar phases, were observed in the silicatesurfactant-rich phase. The model was later extended to investigate ordered hybrid organic-inorganic materials. ${ }^{14,15}$ Later Jin et al. ${ }^{16}$ applied this surfactant model together with a silicate tetrahedral model to study the self-assembly of MCM41 formation, taking into account the silica condensation. A two-step formation mechanism was proposed and investigated: (i) the reversible emergence of mesostructures in silica monomer-surfactant systems, and (ii) the irreversible silica condensation for forming MCM-41.

Lattice models are limited in the level of atomic detail that can be addressed. On the other hand, detailed understanding of the formation pathway by simulations at the atomistic level is restricted by the high complexity and large system size of the self-assembly process. ${ }^{17,18}$ To overcome such limitations, Jorge and co-workers ${ }^{19}$ applied the MARTINI model ${ }^{20,21}$ and developed coarse-grained (CG) silicate parameters to investigate the formation of PMSs. This represents an intermediate degree of coarse graining between the lattice models and fully atomistic models. The authors found that silica monomers induced micelle fusion, promoting a sphere-to-rod transition in the early stages of PMS formation. We recently extended this CG model to describe different silica oligomers in MCM-41 precursor solutions. ${ }^{22}$ Silica oligomers were found to promote the formation of hexagonal structures during the MCM-41 formation in systems with a very low surfactant concentration. This work also suggested that, in the studied systems, silica oligomers are essential to MCM-41 synthesis, and the results supported the CTM pathway of MCM-41 formation under those conditions.

Here we expand our previous work ${ }^{19,22}$ using the MARTINI CG model to study the MCM-41 formation mechanism at high surfactant concentrations. In the original liquid crystal templating hypothesis ${ }^{9}$ it is assumed that silicates would accumulate at the interfaces in a preassembled surfactant-water mesophase structure. We investigate this possibility by performing molecular dynamics simulations with our CG model at conditions under which the surfactant alone can already form structured mesophases in aqueous solution. This is quite distinct from our previous studies at low concentration, where pure surfactant solutions were only able to form micelles. There are two components to our present work. First, we have studied in detail the phase behavior of the surfactant-water system in the context of our model. Our results show quite good qualitative agreement with experiment regarding the surfactant concentrations associated with various liquid crystal phases. We then consider the behavior of systems where silicates are added to states with preformed surfactant-water mesophases. We have discovered that the original hexagonal mesophase structure is initially destroyed by the addition of silica monomers, but is later reformed with the silica dimers concentrating at the surfactant/water interface. Furthermore, our model is also able to describe the formation of surfactantsilica mesostructures that are analogous to other PMS materials, such as MCM-48 and MCM-50. ${ }^{9}$ The remainder of this article is organized as follows: section 2 describes the model and simulation methods used herein, section 3 details the results and discussion, and section 4 summarizes the work and offers concluding remarks.

\section{MODELS AND SIMULATION DETAILS}

All molecular dynamics (MD) simulations were carried out with the Gromacs 4.6.1 package, ${ }^{23-26}$ and the leapfrog algorithm ${ }^{27}$ was adopted to integrate the equations of motion. The temperature was fixed through the velocity-rescaling thermostat $^{28}$ with a time constant of $1.0 \mathrm{ps}$, while the Berendsen pressure coupling method ${ }^{29}$ was used to maintain a pressure of 1 bar with a time constant of 5.0 ps. We carried out tests using extended Lagrangian thermostats and barostats, and found the results to be insensitive to these choices. Periodic boundary conditions with isotropic pressure scaling were used. Integration time steps of $30 \mathrm{fs}$ were used in the simulations, as suggested by the authors of the MARTINI model. ${ }^{20,21}$

Unless otherwise stated, all systems were prepared initially by randomly placing all the molecules into cubic boxes using the Packmol package. ${ }^{30}$ The following procedure was applied for all simulations carried out in this study: (1) use the steepestdescent algorithm to minimize the total energy of the system to prevent short-range contacts between atoms; (2) perform a short (150 ps) simulation in the NVT ensemble to stabilize the kinetic energy under the chosen conditions; (3) carry out production runs in the $N p T$ ensemble with a total simulation time ranging from a few microseconds to tens of microseconds.

The nonbonded interactions, such as Coulombic and van der Waals, were computed with a $1.2 \mathrm{~nm}$ cutoff distance and with the standard Gromacs shift functions for the MARTINI model to mimic the distance-screening phenomena. ${ }^{21}$ The shifting of the potential was from 0.9 to 1.2 and from 0 to $1.2 \mathrm{~nm}$ for van der Waals and Coulomb, respectively. In addition to the nonbonded interactions (i.e., Lennard-Jones and Coulombic terms) described above, bonded interactions including bond stretching, angle bending, and torsion were also taken into account. More details regarding the bonded potential parameters can be found in our previous work. ${ }^{19}$

The MARTINI 2.2 force field was adopted for the CG models with the standard MARTINI dielectric constant value of $15 .^{21}$ Schematic representations of our CG model in this work are shown in Figure 1. The amphiphilic $\mathrm{CTA}^{+}$surfactant is described by four $\mathrm{C}_{1}$ apolar $\mathrm{CG}$ beads for the hydrophobic tail groups and a charged $Q_{0}$ CG bead for the hydrophilic headgroup. Despite its simplicity, this model has been successfully used by ourselves ${ }^{19}$ and others ${ }^{31}$ to describe the self-assembly of cationic ammonium surfactants. Bromide counterions are described as in the original MARTINI model by a $Q_{a}$ bead, which is assumed to include a solvation shell of six water molecules. Four water molecules are represented by a $\mathrm{P}_{4}$ bead, and a $10 \%$ fraction of CG water particles were replaced by antifreeze CG water particles ${ }^{20}$ to avoid the tendency of unrealistic freezing of water in the MARTINI CG model. This antifreeze particle is denoted as $\mathrm{BP}_{4}$. A larger $\sigma$ value and a greater potential well, $\epsilon$, of the Lennard-Jones potential interaction of $\mathrm{BP}_{4}-\mathrm{P}_{4}$ compared to that of $\mathrm{P}_{4}-\mathrm{P}_{4}$ are assigned to disturb the lattice generated by the uniform size of solvent particles and to avoid the phase separation of two different solvent particles. ${ }^{21}$ The interaction of antifreeze particles with other particle types is the same as that of regular CG water particles. An alternative approach to avoid this artificial freezing of water is to use a polarizable coarse-grained water model. ${ }^{32}$ However, this can increase the computational cost by about a factor of 3 over the standard CG water model, which would 

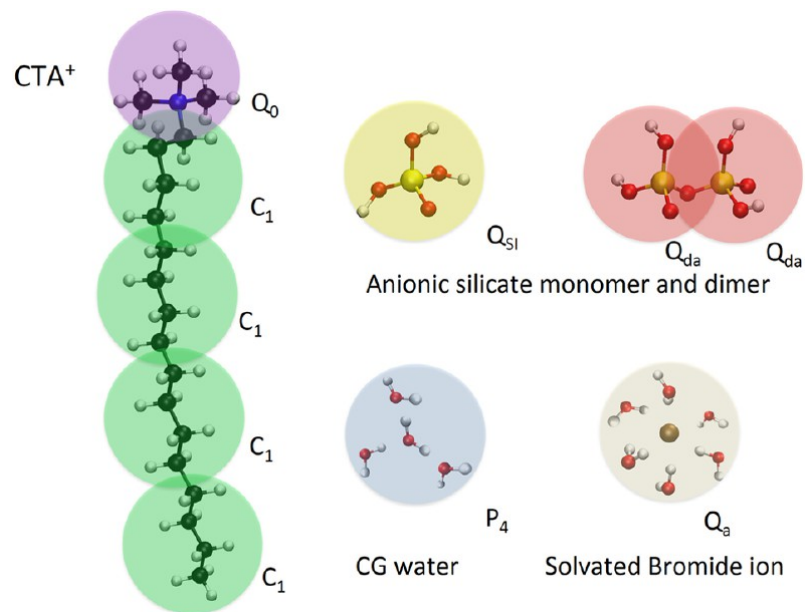

Anionic silicate monomer and dimer
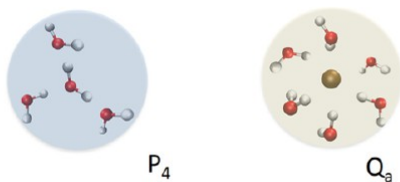

CG water

Solvated Bromide ion

Figure 1. Schematic representations of the coarse-graining procedure employed in this work: $\mathrm{CTA}^{+}$surfactant, singly charged silica monomer, doubly charged silica dimer, solvated bromide ion (implicitly solvated by six water molecules), and water (representing four water molecules). The labels correspond to MARTINI bead types except the $\mathrm{Q}_{S \mathrm{I}}$ particle which was defined in our previous work. ${ }^{1}$

make it prohibitive to analyze the phase diagrams of the system in detail. The anionic silica monomer is described by one $Q_{S I}$ CG bead, ${ }^{19}$ and each silica dimer is represented by two $Q_{d a} C G$ beads. The CG parameters of silicate species were obtained by comparing the density profiles of preshaped spherical micelles in both all-atom and CG simulations. The detailed procedure of CG silicate parametrization can be found in our previous work. $^{22}$

The effective time scale of the MARTINI CG model is generally 2-10 times faster than the time scale of an atomistic model. $^{21}$ To quantify the speedup of the CG model in our specific system, we have computed the self-diffusion coefficient of a single CTAB molecule in water for both CG and all-atom (AA) simulations. The self-diffusion coefficients are $0.7366 \times$ $10^{-5}$ and $2.9096 \times 10^{-5} \mathrm{~cm}^{2} / \mathrm{s}$ for AA and CG simulations, respectively. The latter calculation takes into account the 4:1 mapping of CG beads. ${ }^{20,33}$ This provides an estimated speedup factor of 3.95 for the CG dynamics, although this is likely to change at higher surfactant concentrations, where dense aggregates are formed. For consistency, in the remainder of this article we simply report the actual simulation time for each run.

Different concentrations of $\mathrm{CTA}^{+}$together with bromide ions $\left(\mathrm{Br}^{-}\right)$, anionic silica monomers $\left[\left(\mathrm{SiO}_{4} \mathrm{H}_{3}\right)^{-}\right]$, or doubly charged silica dimers $\left[\left(\mathrm{Si}_{2} \mathrm{O}_{7} \mathrm{H}_{4}\right)^{2-}\right]$ under high $\mathrm{pH}$ conditions were studied in this work. It is well-known that $95 \%$ of silicic acids are deprotonated at $\mathrm{pH}$ as low as $11 ;^{34}$ therefore each silicate molecule in our model holds a single negative charge per silicon atom in all simulations. Various temperatures ranging from 300 to $430 \mathrm{~K}$ were considered. Different mesophase types were identified visually and by calculating structural properties such as density profiles (see the Supporting Information for details). To check for equilibrium, we have monitored the evolution of energy, pressure, and density profiles with time. In several cases, particularly at low temperatures, where equilibrium was more difficult to achieve, we ran simulations starting from different initial conditions (e.g., random and preformed mesostructures), as discussed below. Complete tables with the individual simulation times for each surfactant concentration (given in weight percent surfactant) and temperature are provided in the Supporting Information (Tables S1-S3). Input files for all simulations carried out in this paper are openly available through the University of Strathclyde's data repository at http://dx.doi.org/ 10.15129/cf144cdf-810e-4c12-ab81-5056ef03bd1a.

\section{RESULTS AND DISCUSSION}

3.1. Water-Surfactant System $\left(\mathrm{CTA}^{+}-\mathrm{Br}^{-}-\mathrm{H}_{2} \mathrm{O}\right)$. To examine whether our $\mathrm{CG}$ model can predict the phase behavior of aqueous surfactant solutions, the phase diagram of our model was investigated and compared with experimental observations ${ }^{35}$ as seen in Figure 2. In addition, readers can refer to the schematic phase diagram by Brinker ${ }^{36}$ (see Figure 2 on page 801 in ref 36). Differences between our results and those from experiment reflect the approximations in our model, as discussed in more detail below, and also other factors such as
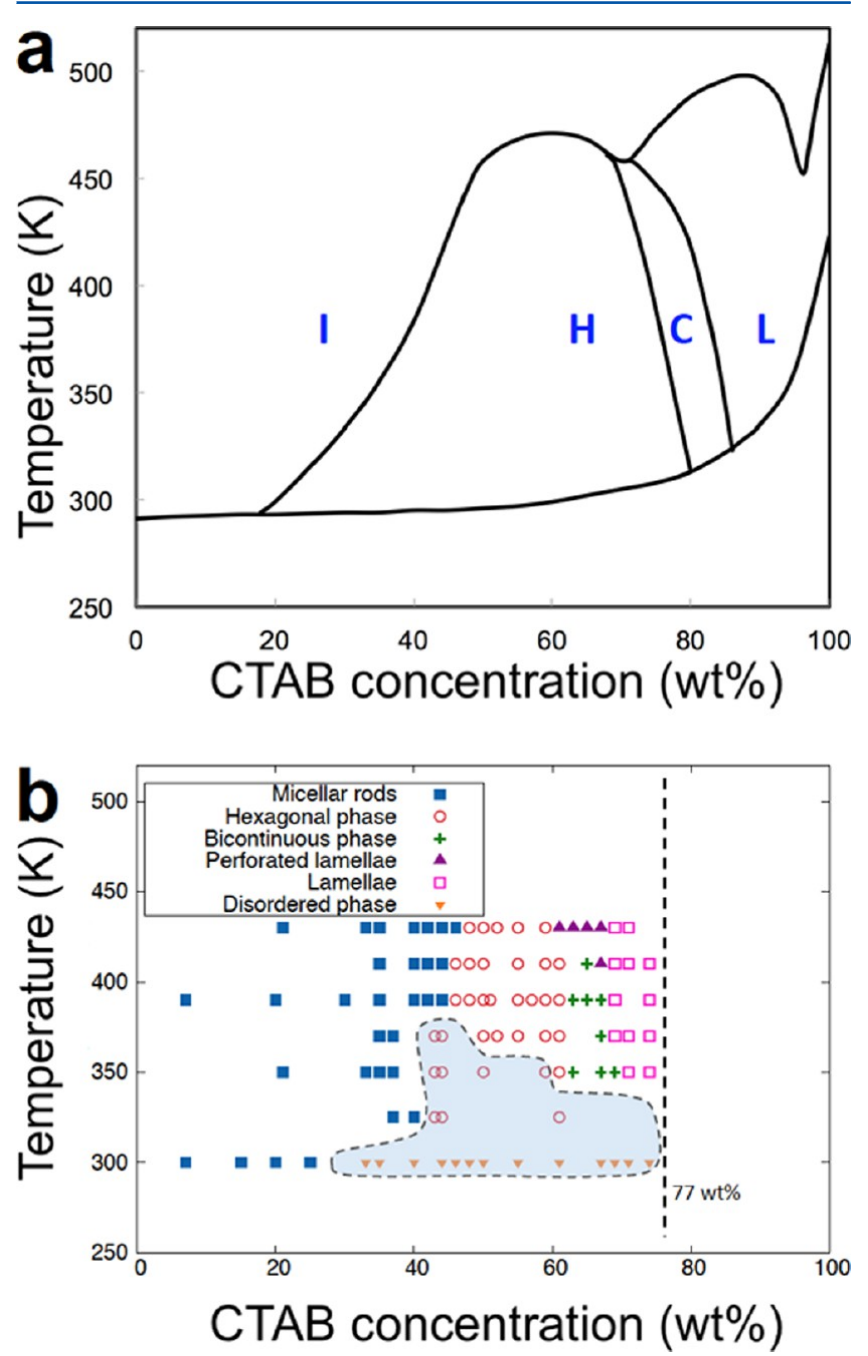

Figure 2. (a) Phase diagram from experimental observations. Replotted based on data extracted from a figure in ref 35. I, isotropic solutions including both spherical micelles and micellar rods; $\mathrm{H}$, hexagonal; C, bicontinuous cubic phase; L, lamellar. (b) Phase diagram from our simulations. Each data point in (b) represents one equilibrated $\mathrm{MD}$ run. The vertical dashed line at $\sim 77$ wt $\%$ surfactant represents the limit accessible by the MARTINI model, and the gray area represents the simulating conditions that yielded kinetically trapped structures from random initial configurations. 


\section{a $\quad 18 \mathrm{wt} \%$}
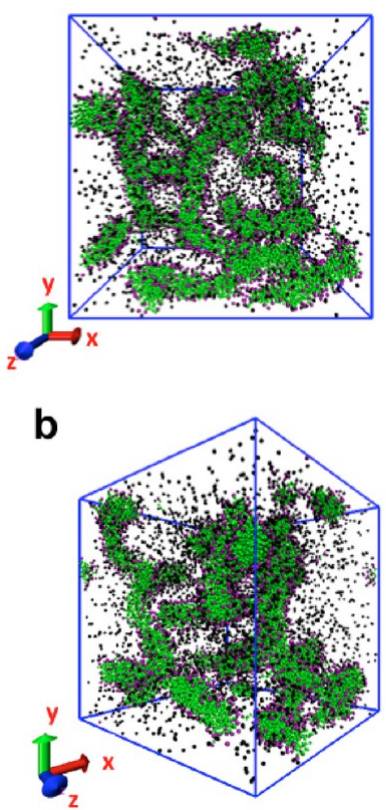

C $55 \mathrm{wt} \%$
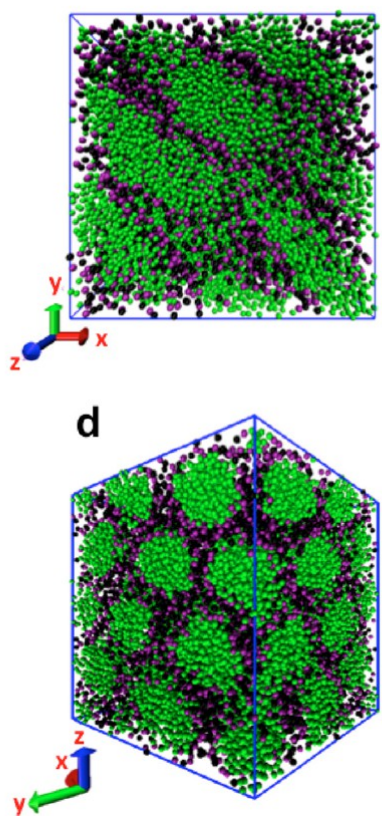

e $74 \mathrm{wt} \%$
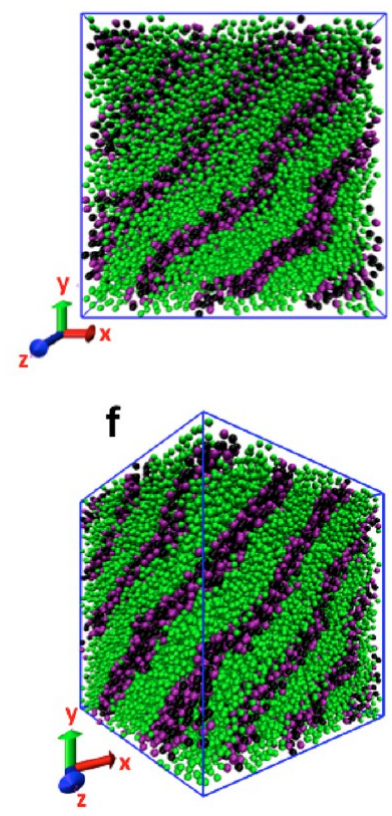

Figure 3. Visualizations of selected mesophases obtained from this work. Two different views are shown for each mesostructure. This figure includes $(\mathrm{a}, \mathrm{b})$ micellar rods $(18 \mathrm{wt} \%),(\mathrm{c}, \mathrm{d})$ the hexagonal array $(55 \mathrm{wt} \%)$, and (e, f) the lamellae $(74 \mathrm{wt} \%)$ at $390 \mathrm{~K}$. Color code: green for surfactant tail groups; purple for surfactant head groups; black for bromide ions (water molecules have been removed for clarity).

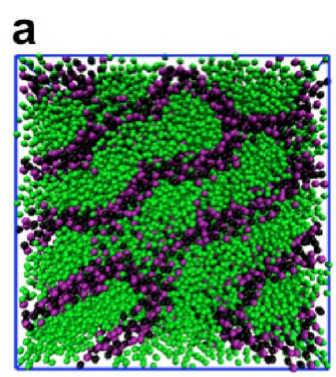

Bicontinuous phase

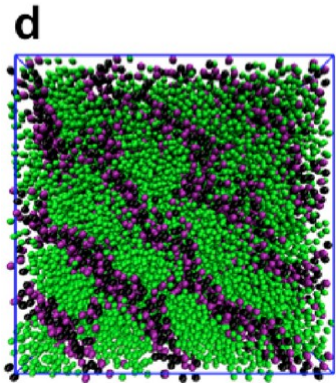

\section{Perforated lamellae}
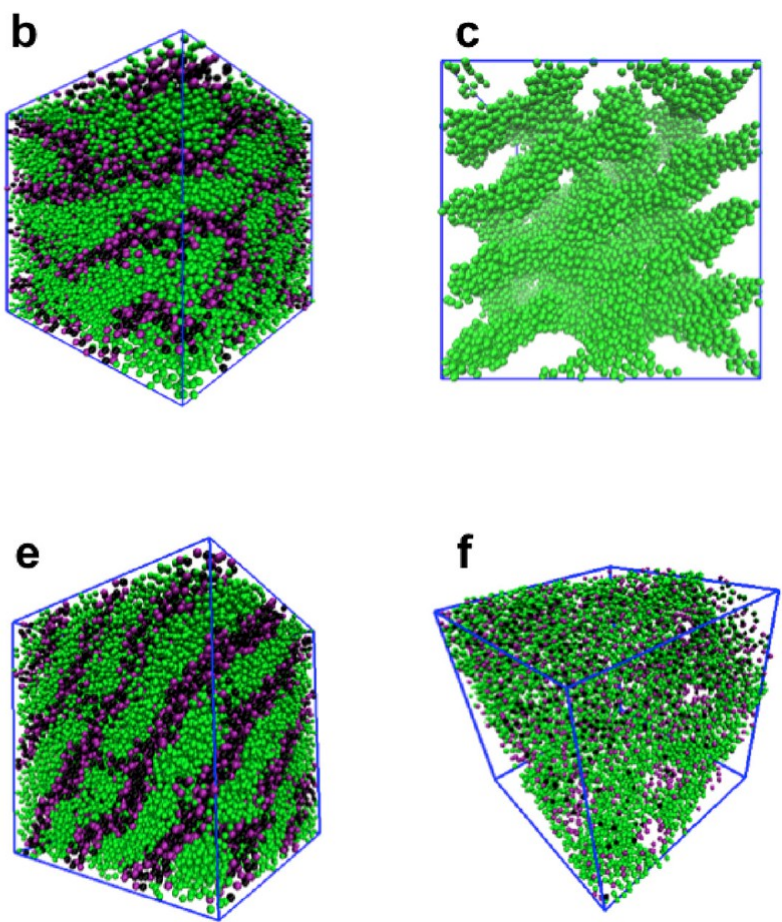

Figure 4. Visualizations of different mesophases obtained from our simulations, including two intermediate phases found with surfactant concentration between those of hexagonal arrays and the lamellar phase: (a)-(c) show different perspectives of a bicontinuous structure (obtained with 67 wt \% CTAB at $390 \mathrm{~K}$ ) and (d)-(f) show perforated lamellae (obtained with 67 wt \% CTAB at $430 \mathrm{~K}$ ). Color code: see Figure 3.

the purity of surfactants used in the experiments. ${ }^{37-39}$ To obtain the simulated phase diagram in Figure $2 b$, a series of simulations were performed with different surfactant concentrations at temperatures ranging from 300 to $430 \mathrm{~K}$. The numbers of $\mathrm{CTA}^{+}$and counterions were both fixed at 4000 , and the number of water molecules was adjusted based on the corresponding concentration of each simulation run. Each point in the plot represents an individual equilibrated simulation run. The gray area shown in Figure $2 \mathrm{~b}$ indicates the systems that yielded kinetically trapped structures starting from random initial configurations. Detailed explanations are provided in subsequent sections of the paper. The corresponding 
mesostructures in this diagram are shown in Figures 3 and 4. In this article we focus on the high surfactant concentration regime, as our previous studies ${ }^{22}$ have already demonstrated the ability of the CG surfactant model to describe the self-assembly of CTAB into spherical and rodlike micelles at low concentrations.

Our simulations show the formation of different mesophases (e.g., micellar rods, hexagonal and lamellar structures) as the surfactant concentration is increased, which is consistent with experimental observations on the phase behavior of CTAB surfactants. Figure 3 shows the visualizations of micellar rods, hexagonal phase, and lamellar phase obtained from our simulations at $390 \mathrm{~K}$ with surfactant concentrations of 18,55 , and $74 \mathrm{wt} \%$, respectively (see the Supporting Information for details on the calculation of weight percent surfactant concentration). At a concentration of $18 \mathrm{wt} \%$, a few micellar rods were obtained-formed by the gradual fusion of spherical micelles as discussed in detail in our previous article. ${ }^{19}$ Figure $3 \mathrm{c}$,d shows the ordered arrangement of the hexagonal array. Evenly distributed cylindrical micelles with a relatively uniform size can be seen. The density profiles for the hexagonal phase show out-of-phase oscillatory peaks for the surfactant heads and tails in two Cartesian directions, while the profiles are flat in the third direction, along the length of the cylindrical micelles (Figure S1 in the Supporting Information). This reveals the two-dimensional periodicity of the hexagonal mesophase. At higher surfactant concentrations, a lamellar phase is obtained that shows an ordered arrangement of bilayers of surfactants. Density profiles for this phase (Figure S2 in the Supporting Information) show regular out-of-phase peaks for heads and tails in one direction, perpendicular to the plane of the bilayers, but are featureless for the other two directions, evidencing the one-dimensional periodicity of the lamellae.

In addition, two different phases at surfactant concentrations in the range between the emergence of hexagonal structure and lamellar phase were found: a bicontinuous phase and perforated lamellae. Figure $4 a-c$ shows the bicontinuous structure found at $390 \mathrm{~K}$ with surfactant concentration of $63-67 \mathrm{wt} \%$ (see Figure 2), slightly greater than the concentration that yields a hexagonal array. In order to have a clearer view of the structure, a snapshot with only the last three tail group beads is shown in Figure $4 c$, as well as an 20 -bead-thick slice of Figure $4 c$, shown in Figure S3 in the Supporting Information. A continuous phase of surfactant tail groups can be seen in the snapshots. This structure corresponds to the cubic phase in the experimental phase diagram in Figure $2 \mathrm{~b}$. Due to the simplified nature of our model, it remains challenging to determine whether the structure obtained in our simulation has a true cubic symmetry. Density profiles for this structure (Figure S4 in the Supporting Information) show pronounced oscillations in all three directions of space, supporting a cubic periodicity. However, the profiles are rather irregular, evidencing the inherently disordered nature of this mesostructure. We thus choose to identify this as a putative bicontinuous phase.

Figure $4 \mathrm{~d}-\mathrm{f}$ shows perforated lamellae obtained in a system with 67 wt \% surfactant at $430 \mathrm{~K}$. The structure emerged within $0.2 \mu$ s and remained after $\sim 10 \mu$ s. Parallel planes with holes and inhomogeneities of the surfactant layers in the lamella can be seen in Figure 4f, where only one lamella is shown for clear visualization. Although not clearly identified in the experimental phase diagram shown in Figure 2a, perforated lamellae have been observed previously in concentrated surfactant solutions. $^{40}$
Going back to Figure 2, it is clear that our model shows very good qualitative agreement with experiment in terms of the overall shape of the phase diagram and the sequence of observed mesophases. Isotropic, hexagonal, bicontinuous, and lamellar phases were observed as the surfactant concentration increases. Quantitative agreement, however, is more challenging to ascertain. Disregarding any uncertainty in the experimental data, discrepancies can be due to at least two possible reasons related to our simulations: (i) difficulty in reaching equilibrium at low temperatures; (ii) limitations of the CG representation. Within the latter, there are several assumptions of the MARTINI model that can affect the results, such as lack of atomistic detail, neglect of hydrogen bonding, and simplified treatment of electrostatic interactions. Decoupling the precise effects of each of these approximations is very challenging, and in some cases not even possible (for example, atomistic simulations of dense surfactant mesophases remain out of reach of current computational resources). Nevertheless, there is one particular assumption of MARTINI that has a direct impact on the phase diagram, and which is relatively easy to quantify. The representation of bromide (and any other small ions) by a single bead containing six solvating water molecules imposes a limit on the highest CTAB concentration that can be simulated. This is represented by a vertical dashed line in Figure $2 b$ at around $77 \mathrm{wt} \%$. As explained in detail in the Supporting Information, even when no CG water beads are included in the simulation, the bromide solvation water means that the surfactant concentration cannot go above this limit. The consequence of this assumption is that the phase diagram appears compressed at high concentrations, relative to the experimental one, and the onset of the high-density phases is shifted to lower concentrations.

To try to assess the impact of the bromide representation in the MARTINI model, we have replotted the phase diagram from Figure $2 \mathrm{~b}$ with surfactant concentrations recalculated without accounting for the solvation water molecules (see the Supporting Information for details). This is equivalent to assuming that the bromide beads represent bare, rather than solvated, ions. The replotted phase diagram, shown in Figure S5 of the Supporting Information, shows much better quantitative agreement with experiment for the regions of stability of the bicontinuous and lamellar phases. However, the boundary between the hexagonal and micellar phases is now shifted to higher concentrations, worsening the agreement with experiment. Taken together, these results suggest that the assumption of the MARTINI model regarding the solvation of bromide ions is suitable at relatively low ion concentrations, but breaks down at high ion concentrations. The most realistic approach would therefore be to consider a gradual loss of bromide solvation waters as the concentration increases. It is beyond the scope of this paper to elaborate further on this possibility, so we retain the original MARTINI assumption in the remainder of the discussion, bearing in mind its inherent limitations.

An additional challenge when comparing simulations to experiments is that the former may not be able to reach equilibrium within a reasonable time scale. Instead, simulations may become trapped in metastable states and the outcome may depend on the starting configuration. Indeed, when we initially probed the phase diagram using single MD runs starting from random initial configurations, we observed that several simulations at temperatures below $390 \mathrm{~K}$ became kinetically trapped, while at $390 \mathrm{~K}$ and above, equilibrium was always 

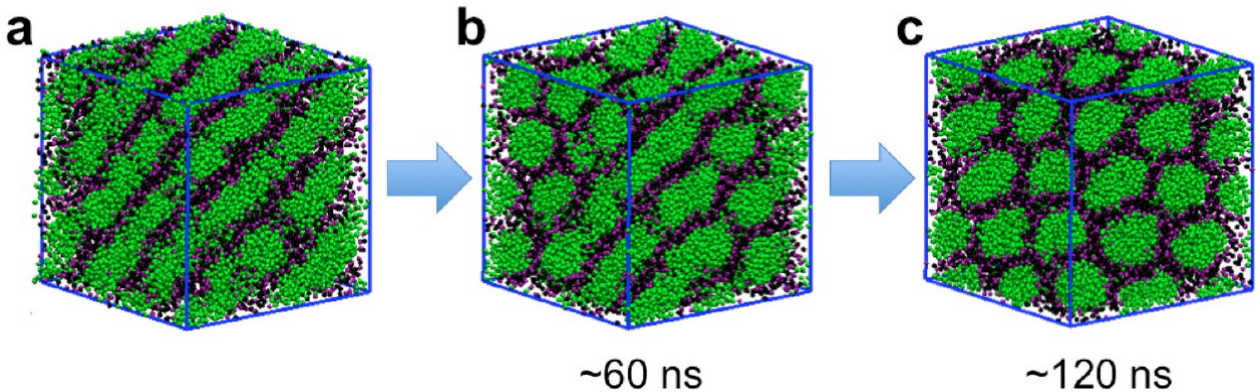

Figure 5. Snapshots of a simulation at $390 \mathrm{~K}$ using perforated bilayers (obtained from a system of 61 wt \% CTAB at $430 \mathrm{~K}$ ) as an initial configuration. Color code: see Figure 3.
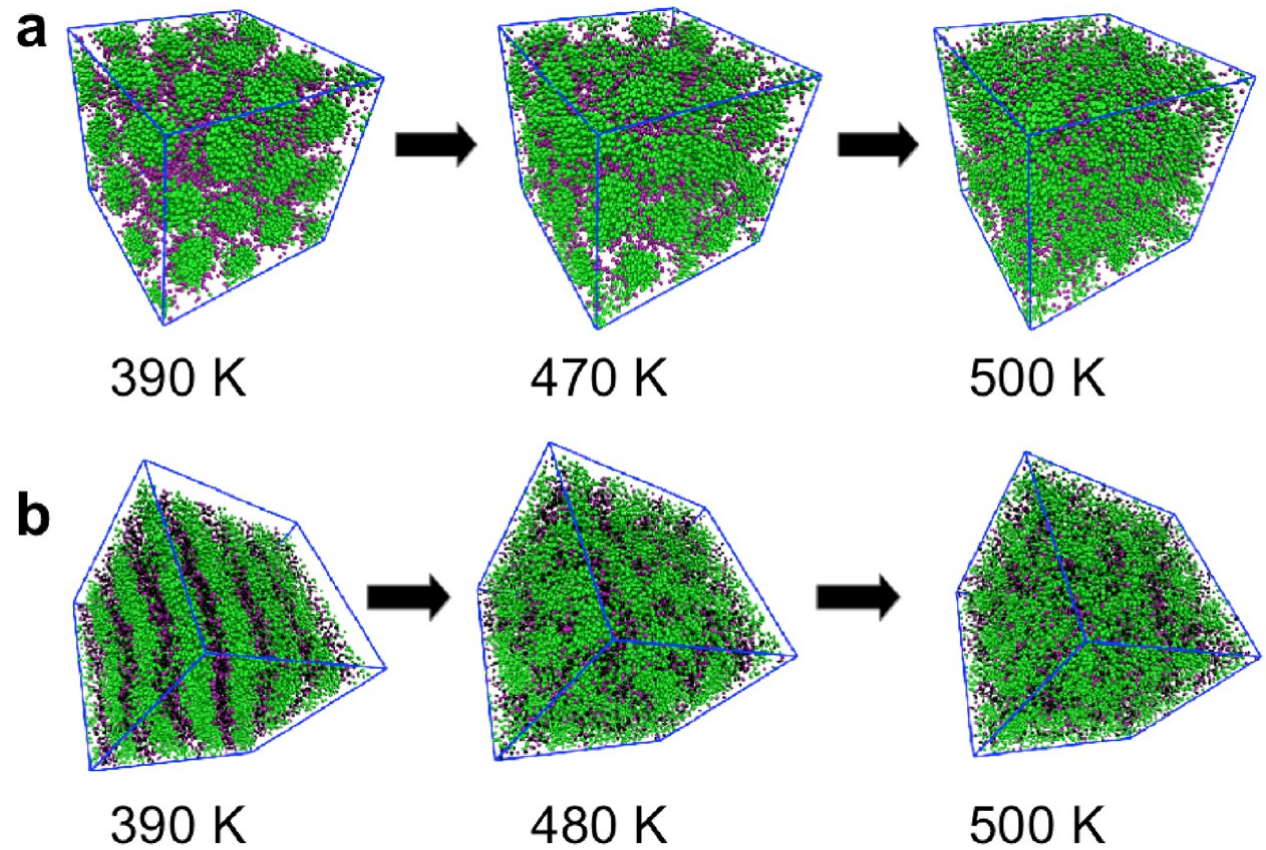

$480 \mathrm{~K}$

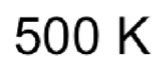

Figure 6. Visualizations of system configurations for the phase change from an initially ordered phase to a disordered phase with the following concentrations of CTAB: (a) 61 and (b) 69 wt \%. Color code: see Figure 3.

reached within a reasonable time. The phase diagram obtained from these initial runs is shown in Figure S6 of the Supporting Information. In what follows, we analyze those two regimes in turn, starting with the high-temperature region of the phase diagram. To better establish the equilibrium states in different regions of the phase diagram, we carried out additional runs starting from preequilibrated mesophases and changing either the temperature, the concentration, or both. In the "concentration-swing" simulations, the surfactant concentration was increased or decreased by removing or adding water molecules to the system, respectively. After each perturbation, the system was allowed to reequilibrate for a long enough period. We then tested the reversibility of the observed transitions by simulating the opposite process (e.g., diluting, then concentrating the system). Although this does not necessarily guarantee that equilibrium is reached, it does lead to much more robust predictions of the ranges of stability of each mesophase.

In the high-temperature region of the phase diagram, simulation runs starting from preformed mesophases generated the same final structures as those that started from random initial configurations. For instance, a simulation performed at $390 \mathrm{~K}$ using preformed perforated bilayers (obtained at $430 \mathrm{~K}$ with $61 \mathrm{wt} \%$ ) as the initial configuration eventually yielded a hexagonal array, consistent with starting from random initial conditions. The evolution of the configuration is shown in Figure 5, depicting the gradual transition from the perforated bilayers to a hexagonal array. All other such tests for temperatures at or above $390 \mathrm{~K}$ strongly suggest that the simulations at these higher temperatures have indeed reached equilibrium.

To test the upper limits of the phase diagram, gradual heating simulations were performed using two different initial configurations: hexagonal and lamellar structures taken from our previous studied simulations. The two systems we studied are as follows: (1) $61 \mathrm{wt} \%$ CTAB (hexagonal) and (2) 69 wt \% $\mathrm{CTAB}$ (lamellar). The temperature was increased from 390 to $500 \mathrm{~K}$ with intermediate steps at 460,470 , and $480 \mathrm{~K}$, with each step having a simulation time of $0.012 \mu$ s. Figure 6 shows how both the initially ordered phases gradually yield a disordered phase at higher temperatures. The phase transition was found to occur around $470 \mathrm{~K}$ in the case of $61 \mathrm{wt} \% \mathrm{CTAB}$ and at 480 $\mathrm{K}$ in the case of $69 \mathrm{wt} \% \mathrm{CTAB}$. Reversibility of the phase transition was confirmed by decreasing the temperature with the same time steps and simulation time. The transition temperatures predicted from our model are in very good 
agreement with the upper boundaries of the experimental phase diagram (see Figure $2 \mathrm{a}$ ).

As mentioned above, at temperatures below $390 \mathrm{~K}$, some simulations showed signs of kinetic trapping. Indeed, the single runs starting from random initial configurations (Figure S6) predicted a much wider region of stability of the micellar phase at low temperature, suggesting a phase boundary with an unrealistic inverted curvature relative to experiment. To help overcome free energy barriers and allow the low temperature systems to reach equilibrium, we attempted to run a replica exchange MD (REMD) simulation ${ }^{41}$ at $50 \mathrm{wt} \%$ surfactant over a temperature range of 300-403 $\mathrm{K}$. To ensure efficient replica exchange moves, 60 replicas were required, which made the simulation extremely computationally demanding. After almost $6 \mu \mathrm{s}$, the simulation had not yet converged, suggesting that a range extending to even higher temperatures is needed to ensure equilibration. Unfortunately, the large computational expense makes it prohibitive to probe the phase diagram in any detail using REMD simulations. As an alternative, we performed several of the "temperature-swing" and "concentration-swing" simulations described above. For example, starting from a hexagonal phase obtained at $390 \mathrm{~K}$ and $46 \mathrm{wt}$ $\%$, we lowered the temperature to $370 \mathrm{~K}$ and added water to obtain a concentration of $44 \mathrm{wt} \%$. After running the simulation for $12 \mu \mathrm{s}$, the hexagonal structure was maintained. As we further decreased the concentration to $43 \mathrm{wt} \%$, the hexagonal structure remained after running for $2.3 \mu \mathrm{s}$. However, when the system was further diluted to a concentration of $37 \mathrm{wt} \%$, the hexagonal phase changed to a disordered assembly of rodlike micelles (see Figure 7), similar to that obtained under the same

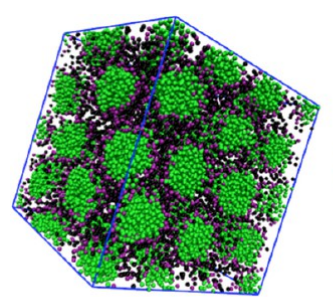

$44 w t \%$

\section{$\stackrel{\begin{array}{c}\text { Adding } \\ \text { water }\end{array}}{\stackrel{\text { Removing }}{\text { water }}}$}

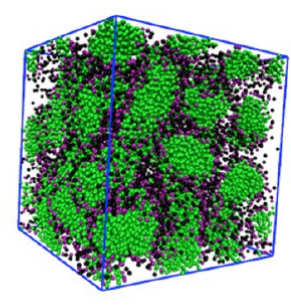

$37 w t \%$
Figure 7. Visualizations of the reversible transition between hexagonal array and micellar rods by adding/removing water molecules between 44 and $37 \mathrm{wt} \%$ at $370 \mathrm{~K}$. conditions but starting from a random initial configuration. This suggests that the boundary between the micellar and hexagonal phases at $370 \mathrm{~K}$ lies somewhere between 37 and 43 wt \%. We also tested the reversibility of this transition by removing water molecules from the micellar configuration at 37 wt $\%$ to obtain a concentration of 44 wt \% (Figure 7). After about $6 \mu$ s of simulation, the hexagonal phase had reformed. This procedure was used for several other temperatures and concentrations, allowing us to obtain a better estimate of the phase transition regions (see Figure $2 \mathrm{~b}$ ). The results show a much more realistic shape of the micellar-to-hexagonal phase boundary, in good agreement with the experimental phase diagram.

The results obtained at $300 \mathrm{~K}$ merit additional discussion. Simulations at high concentration led to a dense packing of micellar rods with no evident long-range order, which remained stable for very long simulation times. This is very likely to be a kinetically arrested phase, which we have denoted simply as a "disordered" phase in the simulated phase diagrams (Figure $2 \mathrm{~b}$, Figures S5 and S6). In fact, this temperature is already below the solid-liquid transition (lower boundary in Figure 2a) at high concentrations, and therefore the kinetically arrested phases we observe in our room temperature simulations are likely to represent metastable states prior to the onset of a solid phase. Indeed, simulations starting from preformed mesostructures obtained at higher temperature (e.g., a hexagonal array from 61 wt \% CTAB and a lamellar phase from 69 wt \% $\mathrm{CTAB}$ ) retained those mesostructures over long simulation times. Even at relatively low concentrations, we were unable to observe reversible transitions between the micellar and the hexagonal mesophase regions. The only points which we are confident enough to assign to a particular phase are those at concentrations below $25 \mathrm{wt} \%$ (both random and preformed initial structures led to the formation of rodlike micelles).

From the study of the simulated phase diagram of CTAB solutions, we have shown that different mesophases can be obtained at the temperature of $390 \mathrm{~K}$ from our simulations within a tractable simulation time scale, and that the final configurations were verified to be the equilibrium states. In addition, systems at this temperature showed very good agreement with experimental observations on the phase behavior. As a result, the temperature was fixed at $390 \mathrm{~K}$ in the subsequent simulations for studying the effect of silica on the formation of PMS. The temperature chosen here is higher than the temperature used in our previous work $(300 \mathrm{~K})$ where

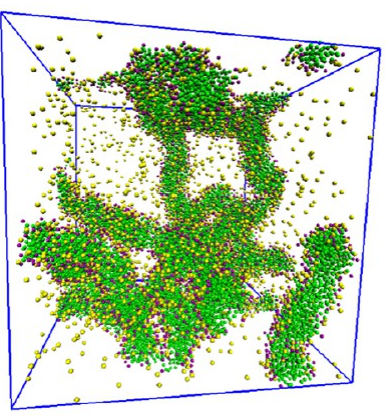

11 wt\%

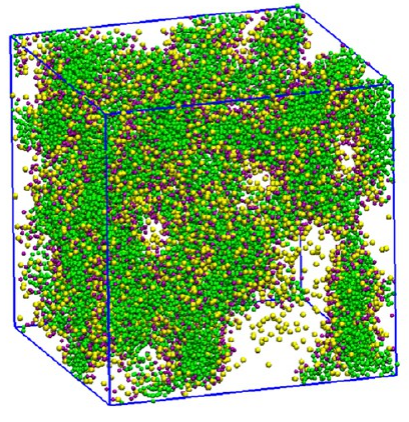

$28 w t \%$

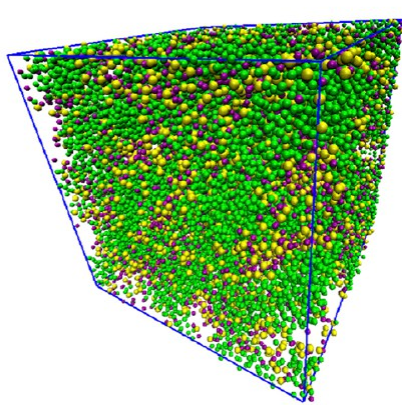

44 wt\%

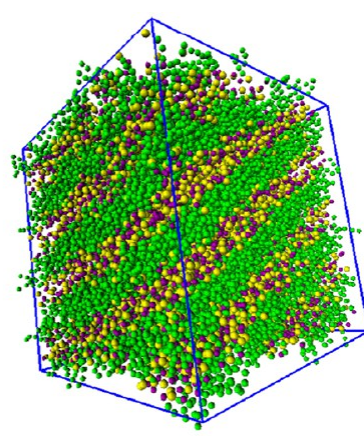

$60 w t \%$

Figure 8. Visualizations of phases obtained in the $\mathrm{CTA}^{+}-\mathrm{SI}-\mathrm{H}_{2} \mathrm{O}$ system at $390 \mathrm{~K}$ with surfactant concentrations of $11,28,44$, and 60 wt \%. Color code: green for surfactant tail groups; purple for surfactant head groups; yellow for silica monomers (water molecules have been removed for clarity). 
a $\mathrm{MCM}-41(30 \mathrm{wt} \%)$
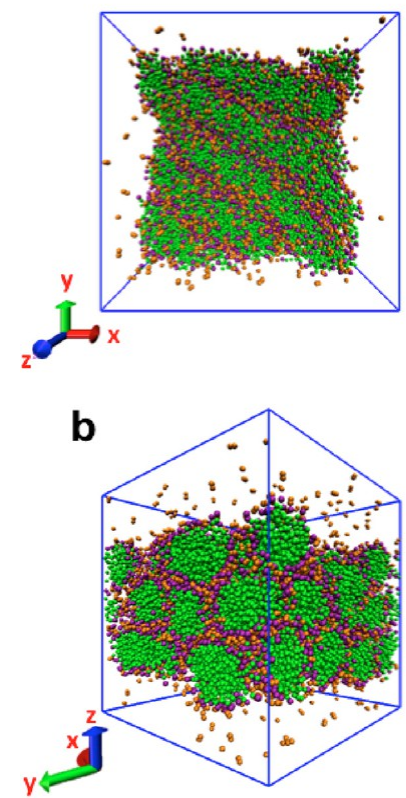

C $\mathrm{MCM}-48(59 \mathrm{wt} \%)$
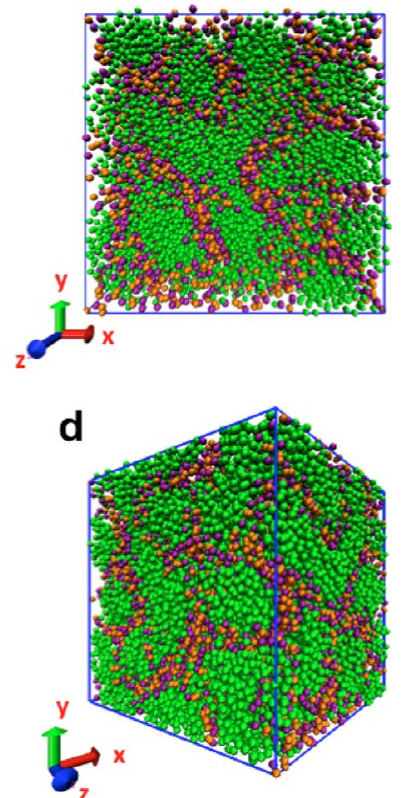

e $\operatorname{MCM}-50(74 \mathrm{wt} \%)$
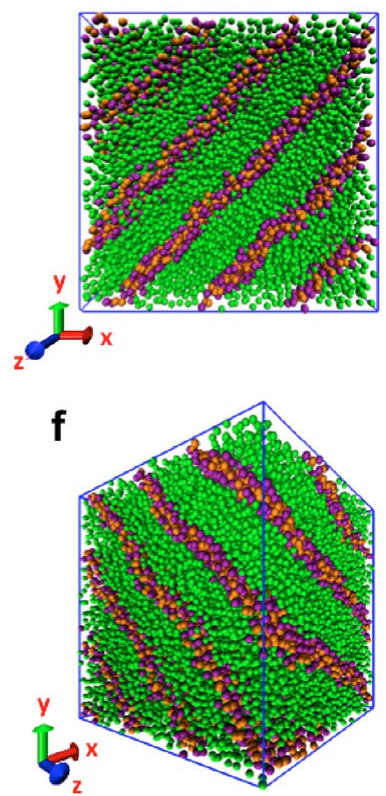

Figure 9. Two different perspectives of phases formed in simulations obtained in the $\mathrm{CTA}^{+}-\mathrm{SI} 2-\mathrm{H}_{2} \mathrm{O}$ system at $390 \mathrm{~K}$ with surfactant concentrations of (a) and (b) $30 \mathrm{wt} \%$, (c) and (d) $59 \mathrm{wt} \%$, and (e) and (f) $74 \mathrm{wt} \%$. Color code: green for surfactant tail groups; purple for surfactant head groups; orange for silica dimers (water molecules are removed for clarity).

we studied the role of the silicates during MCM-41 formation in a low surfactant concentration system. In that extremely low surfactant concentration region, the system is not trapped at a local energy minimum, allowing simulated generation of spherical micelles at $300 \mathrm{~K}$ as predicted by experiment. ${ }^{19,22}$

\subsection{Effect of Inorganic Species: Silica Monomers and} Dimers. In order to study the MCM-41 formation mechanism at high $\mathrm{pH}$, charged silica monomers (SI) were introduced into the $\mathrm{CTAB} /$ water (W) systems studied above. A complete silicate-bromide ion exchange was assumed, consistent with our previous work on this system. ${ }^{17,19}$ Given that each monomer holds a negative charge, equal amounts of monomers and surfactants were used to maintain the charge neutrality of the solution. Figure 8 shows visualizations of a silica monomer system at different surfactant concentrations for $390 \mathrm{~K}$. We note that, as the surfactant concentration was increased, we observed micellar rods, a bicontinuous structure, and then a lamellar phase. However, no hexagonal phase was found from this system over a wide range of surfactant concentrations. Several simulations with silica monomers at temperatures of 300 and $350 \mathrm{~K}$ with surfactant concentrations ranging from 10 to 71 wt \% were performed, and in all cases we did not observe a hexagonal phase. These results suggest that, in the initial stages of MCM-41 formation from CTAB/silicate solutions, silica monomers alone cannot promote the formation of a hexagonal mesophase even in a concentrated surfactant solution. This result extends the findings from Firouzi et al. ${ }^{42}$ and our previous work, ${ }^{22}$ in which both experimental and simulation results showed that a system containing predominantly monomeric silicate anions yielded no hexagonal array in a very low surfactant concentration solution at high $\mathrm{pH}$. Our present work extends this finding to much higher surfactant concentrations.

Similar procedures were applied for silica dimers (SI2). Since each silica dimer possesses two negative charges (one per $\mathrm{Si}$ atom), a dimer: $\mathrm{CTA}^{+}$ratio of 1:2 was applied to maintain the charge neutrality of the system. In contrast to the systems of $\mathrm{CTAB} / \mathrm{W}$ and CTA/W/monomer studied previously, we observed a phase separation between a solvent-rich phase and a silicate-surfactant-rich phase. More importantly, the MCM41 hexagonal structure was also observed in the silica dimer system, in contrast to the silica monomer system. Figure 9 shows the visualizations of different mesostructures obtained from various surfactant concentrations at $390 \mathrm{~K}$, while the corresponding density profiles are shown in Figures S7-S9 of the Supporting Information. These mesostructures correspond to different materials of the M41S family, such as MCM-41, 9,10 MCM-48, ${ }^{9}$ and MCM-50. ${ }^{9}$ In particular, a material with MCM41 mesostructure was found at the concentration of $30 \mathrm{wt} \%$, a bicontinuous structure corresponding to MCM-48 was found at the higher concentration of $59 \mathrm{wt} \%$, and a lamellar phase corresponding to MCM-50 was observed at a concentration of 74 wt \%.

It is clear in Figure 9 that the hexagonal structure formed by phase separation at a concentration of $30 \mathrm{wt} \%$ is very different from the hexagonal array formed in the $\mathrm{CTAB} / \mathrm{W}$ system at a high surfactant concentration shown previously in our simulations (Figures 3c,d). In the latter case, the hexagonal structures fill the entire simulation cell. The CTAB/W system is recognized as being in the weak-screening limit, where electrostatic repulsion between micelles predominates and leads to the formation of a space-filling single lyotropic liquid crystal (LLC) hexagonal phase. ${ }^{42}$ In contrast, the CTA/W/ silica dimer system has a higher water content but still exhibits a concentrated hexagonal mesophase in the center of the simulation cell. As discussed in detail in our previous article, ${ }^{22}$ the silica dimers act as bridges between adjacent micelles, strongly screening their repulsive electrostatic interactions and promoting micelle aggregation. The two negative charges on silica dimers are located at the farther extremes of the molecule due to charge repulsion. ${ }^{43}$ Each of those charges interacts with the surface of a different micellar rod, pulling them together 
and promoting the formation of the mesostructure. In addition, according to the "strong-screening limit", the higher charge density of anionic silica dimers more effectively screens the electrostatic repulsions between micelles, and leads to the dominance of dispersion attractions between micelles, thus inducing phase separation. ${ }^{22}$

The results shown here are different from those obtained by Lin et al., ${ }^{16}$ who used a rigid silicate tetrahedral model together with lattice Monte Carlo simulations to study the MCM-41 formation. Their work suggested that silica monomers promote the mesostructure formation at a high $\mathrm{pH}$ system. A possible explanation of this discrepancy may lie in the low coordination number of the silicate tetrahedron in the lattice model of Lin et al. The interaction site in their lattice model has a greater interaction area and allows for a higher probability of silicatesurfactant interactions than that of the off-lattice model used in this work. In other words, the tetrahedral silica lattice model may actually be mimicking a higher degree of condensation of silicates, such as silica dimers or trimers instead of monomeric silicates. More work is needed to understand the mapping between such on- and off-lattice models.

Figure 10 summarizes the various mesophases found in different systems obtained from this work at $390 \mathrm{~K}$. Comparing
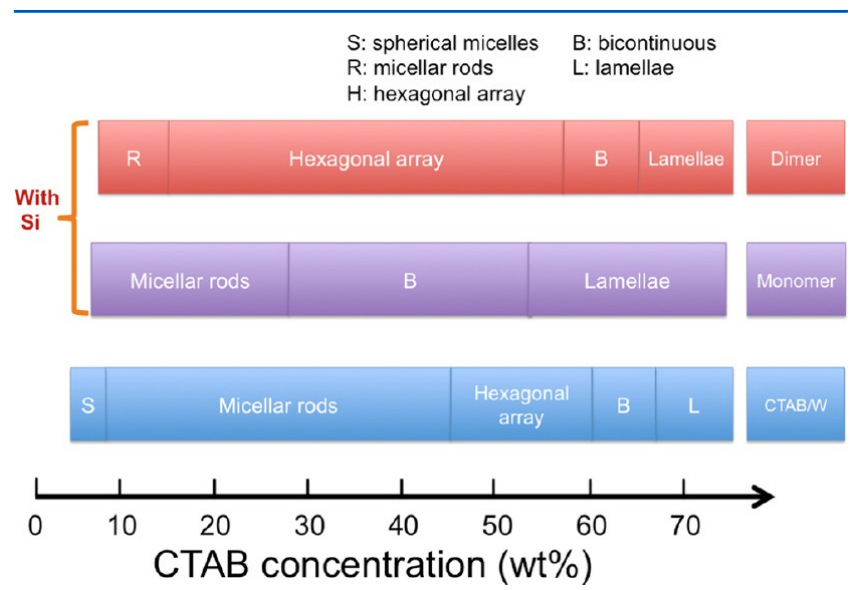

Figure 10. Comparison of the mesophases formed in different systems at $390 \mathrm{~K}$ obtained from this work. Concentrations are all based on the pure surfactant system. the monomer system with the CTAB/W system, it is clear that the regions of stability of the bicontinuous and lamellar phases are significantly extended at the expense of the hexagonal phase. We believe this is related to the very strong adsorption of silica monomers at the surface of surfactant micelles. In fact, those molecules were shown to preferably reside within the headgroup region of the micelles. ${ }^{17}$ This strong adsorption was the driving force for the sphere-to-rod transition observed at low concentrations in our previous work. ${ }^{19}$ It will therefore favor the formation of aggregates with low curvature, such as elongated rodlike structures and lamellae, as observed in the middle panel of Figure 10. Silica dimers, in contrast, adsorb outside the headgroup layer ${ }^{18}$ and hence promote the formation of hexagonal arrays, as described in detail above.

It is clear that the hexagonal structure regions in both $\mathrm{CTAB} / \mathrm{W}$ and $\mathrm{CTA} / \mathrm{W} /$ dimer phase diagrams overlap at surfactant concentrations between 45 and 56 wt \%. It can be inferred from Figure 10 that during the MCM-41 synthesis with a high surfactant content, the hexagonal liquid crystal (HLC) forms at first and then collapses as the silica monomers are added. The hexagonal array would form again as silica begins to oligomerize. As a result, the LCT mechanism of MCM-41 does not appear to be consistent with our simulations. In order to test this hypothesis, we performed simulations at a concentration of $50 \mathrm{wt} \%$ in which the counterions were progressively replaced from bromide to monomers to dimers. We subsequently carried out the reverse trajectory, to confirm that the transitions were reversible (hence taking place at equilibrium). Figure 11 shows the results of this simulation process. A preformed hexagonal array obtained from a CTABwater system after $6 \mu \mathrm{s}$ of MD time is shown in Figure 11a. The bromide ions were directly replaced by silica monomers assuming that complete ion exchange between bromide ions and silica monomers occurs. ${ }^{17,44} \mathrm{~A}$ bicontinuous mesostructure was obtained within $6 \mu \mathrm{s}$ of MD time (Figure 11b). To model the effect of silica dimerization, the silica monomers were then replaced by silica dimers as follows: the water and silica monomers were removed, and silica dimers were added, followed by resolvating the system with the same amount of CG water using Packmol. ${ }^{30}$ A hexagonally ordered arrangement of the micellar rods appeared after $\sim 6 \mu \mathrm{s}$ of $\mathrm{MD}$ time (see Figure 11c). Note that the hexagonal arrays formed in pure $\mathrm{CTAB}$ solutions and in the silica dimer system are significantly different. As previously discussed, the hexagonal structure

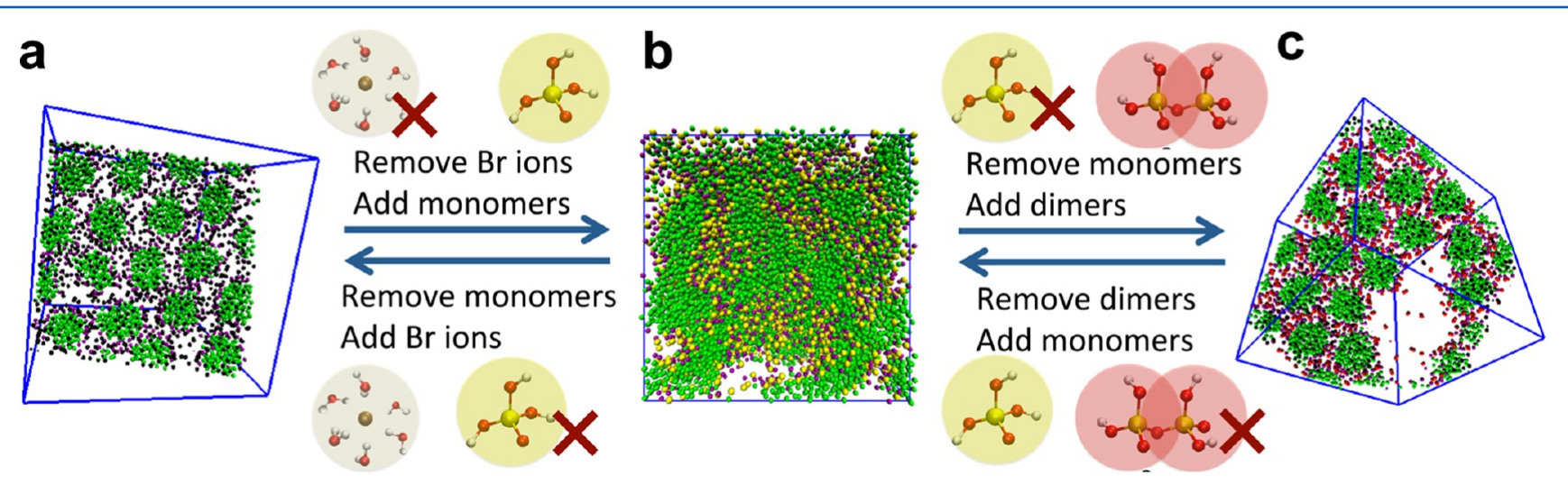

Figure 11. Simulation snapshots obtained in (a) a CTAB solution leading to HLC formation, (b) a solution obtained by replacing all bromide ions by silica monomers, and (c) a solution obtained by replacing all silica monomers by silica dimers. Here we show an $\sim 2-\mu$ m-thick slice of the mesostructure in (a) and (c) for clear visualization. Color code is the same as in Figure 9 (water molecules are removed for clarity). 
formed in the latter case showed a phase separation as seen in Figure $11 \mathrm{c}$, in which the void is representing the position of water molecules. Snapshots of water molecules only for Figure 11 a,c can be seen in Figure S10 in the Supporting Information. An ordered hexagonal structure was found to span the entire simulation box in the pure CTAB solution (Figure S10a); however, the ordered hexagonal structure was only found in the solvent-rich region of the CTA/W/dimer system (Figure $\mathrm{S} 10 \mathrm{~b})$. The opposite process, also represented in Figure 11, showed that this phase transition was indeed reversible.

A similar observation can be made regarding the bicontinuous phase. Although this mesostructure was now observed to be stable with silica monomers, it did so at much lower surfactant concentrations than in the reference CTAB/W system or in the system with silica dimers (see Figure 10). This also suggests that, during the formation of MCM-48 at high concentrations (e.g., between 60 and 70 wt \% surfactant), the bicontinuous phase may be destroyed by the addition of monomers and later reformed after further silica condensation. The lack of overlap observed between the regions of stability of the bicontinuous phase in the three systems studied here may also imply that the LCT mechanism is not viable for MCM-48 synthesis. The exception to this trend seems to be the lamellar phases, which are precursors to MCM-50. At very high surfactant concentrations, these phases are stable with bromide, silica monomers, and silica dimers, suggesting that the LCT mechanism may be feasible under these conditions.

In our earlier simulation work ${ }^{22}$ we found a gradual shift between a hexagonal and a lamellar structure as the degree of silica condensation changed between dimers and octamers. Similar changes in the regions of stability of the mesophases are expected for the top (red) panel in Figure 10 as the silica condensation progresses during the synthesis. Probing in detail the entire phase diagram for different degrees of silica condensation would be too computationally expensive. However, our previous studies at low concentration ${ }^{22}$ showed that silica dimers already capture the essence of the physics of larger silica oligomers. In particular, they are sufficient to promote the phase separation of the system into a dilute solution and an ordered silica-surfactant liquid crystal, as observed experimentally. We thus expect our conclusions about the synthesis mechanism to remain valid for solutions with larger silica oligomers.

In summary, the simulations reported herein show that the system with monomeric silicates (i.e., a high $\mathrm{pH} \sim 14$ system) yields no hexagonal (MCM-41) structure. With the addition of oligomeric silicates, more specifically silica dimers in this work, MCM-41 was found to form. The simulated formation proceeds as follows: at the very initial stages of MCM-41 synthesis, the hexagonal array formed in a pure surfactant solution does not remain intact after the addition of silica monomers. As silica monomers proceed to oligomerize, a hexagonal array forms again. This indicates that some degree of silica condensation is necessary for MCM-41 mesostructure formation. The results also agree with the findings presented by Chen et al., ${ }^{45}$ who carried out the MCM-41 synthesis at surfactant concentrations of 38 wt \% and characterized the selfassembly process using nuclear magnetic resonance (NMR) spectroscopy and X-ray diffraction (XRD) spectroscopy. ${ }^{14} \mathrm{~N}$ NMR showed that the HLC phase was found at $22{ }^{\circ} \mathrm{C}$ before adding a silica source. However, this phase did not survive upon heating up to $95^{\circ} \mathrm{C}$. Therefore, it was suggested by the authors that formation of MCM-41 through the LCT mechanism was not viable. They proposed that randomly ordered rodlike surfactant micelles interact with silicate species and yield approximately two or three monolayers of silicates encapsulating the surface of the MCM-41 mesostructure.

\section{SUMMARY AND CONCLUSIONS}

In the present article, we extended our previous work, elucidating MCM-41 formation at low surfactant concentration, to further investigate the formation mechanism in systems with higher surfactant concentrations. We have investigated whether or not the liquid crystal templating (LCT) formation mechanism is consistent with our simulation data, even in systems where hexagonal phases form before silica addition. The LCT posits the initial formation of surfactant hexagonal structure, around which silica coats and fills in holes prior to silica polymerization. To investigate the validity of the LCT, the phase behavior of CTAB solutions was obtained, and the results showed good agreement with experimental observations. Most importantly, our model at high $\mathrm{pH}$ and high surfactant concentration suggests that MCM-41 forms via the cooperative templating mechanism (CTM) and not the LCT mechanism. The hexagonal liquid crystal in pure CTAB solution was found to collapse as silica monomers were added. With an increasing degree of condensation, silica oligomers form and promote the formation of a phase-separated hexagonal structure. Our results support the CTM: the mesostructure of MCM-41 is determined by the interactions between cationic surfactants and anionic silica oligomers rather than being determined by the pure-surfactant HLC in a high surfactant concentration system. Our results also suggest that the LCT mechanism is not compatible with the synthesis of MCM-48 materials; however, we cannot rule out this mechanism in the synthesis of MCM-50 at very high surfactant concentrations.

Our work shows that the MARTINI CG model is capable of capturing the behavior of organic and inorganic species in solutions while forming different mesostructures at a significant level. The simulations can be carried out for states with an assigned number of oligomers and charges, which cannot be directly accessed by experiments. With a precise control of the silica source in solutions, different materials can be achieved (e.g., MCM-41, MCM-48, and MCM-50). This model exhibits great potential to predict the formation of different mesostructures, leading to a powerful tool for tailoring PMSs. We anticipate that, with the explicit consideration of silica condensation events in the simulation, additional insights into mesoporous materials formation can be achieved in the near future.

\section{ASSOCIATED CONTENT}

\section{S Supporting Information}

The Supporting Information is available free of charge on the ACS Publications website at DOI: 10.1021/acs.jpcc.6b09429.

Additional material as discussed in the text, including simulation details, surfactant/water systems, and surfactant/silica/water systems (PDF)

\section{AUTHOR INFORMATION}

\section{Corresponding Authors}

*E-mail: miguel.jorge@strath.ac.uk.

*E-mail: auerbach@chem.umass.edu.

*E-mail: monson@ecs.umass.edu. 


\section{ORCID}

Szu-Chia Chien: 0000-0002-1239-7258

José R. B. Gomes: 0000-0001-5993-1385

Miguel Jorge: 0000-0003-3009-4725

\section{Present Address}

S.-C.C.: Department of Materials Science and Engineering, The Ohio State University, 2041 N. College Rd., Columbus, OH 43210, USA.

\section{Notes}

The authors declare no competing financial interest.

\section{ACKNOWLEDGMENTS}

P.A.M., S.M.A., and S.-C.C. acknowledge a grant from the U.S. Department of Energy (Contract No. DE-FG02-07ER46466) and the computational resources provided by the Massachusetts Green High-Performance Computing Center (MGHPCC). G.P.-S., M.J., and J.R.B.G. thank the financial support of the PTDC/QUI-QUI/109914/2009 project. This work was developed within the scope of the projects CICECO-Aveiro Institute of Materials, POCI-01-0145-FEDER-007679 (FCT UID/CTM/50011/2013), LAQV@REQUIMTE, POCI/01/ 0145/FEDER/007265, (FCT UID/QUI/50006/2013 and NORTE-01-0145-FEDER-000011), and LSRE (FCT UID/ EQU/500230/2013), financed by national funds through the FCT/MEC and when appropriate cofinanced by FEDER under the PT2020 Partnership Agreement. G.P.-S. acknowledges CICECO-UID/CTM/50011/2013 for grant ref. BI/UI89/ 7145/2015. M.J. acknowledges funding from the EPSRC UK Project Grant EP/L014297/1. Results were partially obtained using the EPSRC funded ARCHIE-WeSt High Performance Computing Centre (www.archie-west.ac.uk and EPSRC Grant $\mathrm{EP} / \mathrm{K} 000586 / 1)$. This work was produced with the support of the Portuguese National Distributed Computing Infrastructure (INGRID). More information in http://www.incd.pt.

\section{REFERENCES}

(1) Corma, A. From Microporous to Mesoporous Molecular Sieve Materials and Their Use in Catalysis. Chem. Rev. 1997, 97, 23732420 .

(2) Kresge, C. T.; Roth, W. J. The discovery of mesoporous molecular sieves from the twenty year perspective. Chem. Soc. Rev. 2013, 42, 3663-3670.

(3) Kruk, M.; Jaroniec, M.; Shin, H. J.; Ryoo, R. In Nanoporous Materials IV Proceedings of the 4th International Symposium on Nanoporous Materials; Sayari, A., Jaroniec, M., Eds.; Studies in Surface Science and Catalysis 156; Elsevier: 2005; pp 55-62.

(4) Kumar, D.; Schumacher, K.; du Fresne von Hohenesche, C.; Grün, M.; Unger, K. MCM-41, MCM-48 and related mesoporous adsorbents: their synthesis and characterisation. Colloids Surf., A 2001, 187-188, 109-116

(5) Ryoo, R.; Jun, S. Improvement of Hydrothermal Stability of MCM-41 Using Salt Effects during the Crystallization Process. J. Phys. Chem. B 1997, 101, 317-320.

(6) Van Der Voort, P.; Mathieu, M.; Mees, F.; Vansant, E. F. Synthesis of High-Quality MCM-48 and MCM-41 by Means of the GEMINI Surfactant Method. J. Phys. Chem. B 1998, 102, 8847-8851.

(7) Monnier, A.; Schuth, F.; Huo, Q.; Kumar, D.; Margolese, D.; Maxwell, R. S.; Stucky, G. D.; Krishnamurty, M.; Petroff, P.; Firouzi, A.; Janicke, M.; Chmelka, B. F. Cooperative Formation of InorganicOrganic Interfaces in the Synthesis of Silicate Mesostructures. Science 1993, 261, 1299-1303.

(8) Firouzi, A.; Kumar, D.; Bull, L.; Besier, T.; Sieger, P.; Huo, Q.; Walker, S.; Zasadzinski, J.; Glinka, C.; Nicol, J.; Margolese, D.; Stucky, G.; Chmelka, B. Cooperative organization of inorganic-surfactant and biomimetic assemblies. Science 1995, 267, 1138-1143.
(9) Beck, J. S.; Vartuli, J. C.; Roth, W. J.; Leonowicz, M. E.; Kresge, C. T.; Schmitt, K. D.; Chu, C. T.-W.; Olson, D. H.; Sheppard, E. W.; McCullen, S. B.; Higgins, J. B.; Schlenker, J. L. A New Family of Mesoporous Molecular Sieves Prepared with Liquid Crystal Templates. J. Am. Chem. Soc. 1992, 114, 10834-10843.

(10) Kresge, C. T.; Leonowicz, M. E.; Roth, W. J.; Vartuli, J. C.; Beck, J. S. Ordered Mesoporous Molecular Sieves Synthesized by LiquidCrystal Templates Mechanism. Nature 1992, 359, 710-712.

(11) Larson, R. G.; Scriven, L. E.; Davis, H. T. Monte Carlo simulation of model amphiphile-oil-water systems. J. Chem. Phys. 1985, $83,2411-2420$.

(12) Siperstein, F. R; Gubbins, K. E. Synthesis and Characterization of Templated Mesoporous Materials Using Molecular Simulation. Mol. Simul. 2001, 27, 339-352.

(13) Siperstein, F. R.; Gubbins, K. E. Phase separation and liquid crystal self-assembly in surfactant-inorganic-solvent systems. Langmuir 2003, 19, 2049-2057.

(14) Patti, A.; Siperstein, F. R.; Mackie, A. D. Phase behavior of model surfactants in the presence of hybrid particles. J. Phys. Chem. C 2007, 111, 16035-16044.

(15) Patti, A.; Mackie, A. D.; Siperstein, F. R. Monte Carlo simulation of self-assembled ordered hybrid materials. Langmuir 2007, 23, 6771-6780.

(16) Jin, L.; Auerbach, S. M.; Monson, P. A. Simulating the formation of surfactant-templated mesoporous silica materials: a model with both surfactant self-assembly and silica polymerization. Langmuir 2013, 29, $766-780$.

(17) Jorge, M.; Gomes, J. R. B.; Cordeiro, M. N. D. S.; Seaton, N. A. Molecular Simulation of Silica/Surfactant Self-Assembly in the Synthesis of Periodic Mesoporous Silicas. J. Am. Chem. Soc. 2007, 129, 15414-15415.

(18) Jorge, M.; Gomes, J. R. B.; Cordeiro, M. N. D. S.; Seaton, N. A. Molecular Dynamics Simulation of the Early Stages of the Synthesis of Periodic Mesoporous Silica. J. Phys. Chem. B 2009, 113, 708-718.

(19) Pérez-Sánchez, G.; Gomes, J. R. B.; Jorge, M. Modeling SelfAssembly of Silica/Surfactant Mesostructures in the Templated Synthesis of Nanoporous Solids. Langmuir 2013, 29, 2387-2396.

(20) Marrink, S. J.; deVries, A. H.; Mark, A. E. Coarse Grained Model for Semiquantitative Lipid Simulations. J. Phys. Chem. B 2004, 108, $750-760$.

(21) Marrink, S. J.; Risselada, H. J.; Yefimov, S.; Tieleman, D. P.; de Vries, A. H. The MARTINI Force Field: Coarse Grained Model for Biomolecular Simulations. J. Phys. Chem. B 2007, 111, 7812-7824.

(22) Pérez-Sánchez, G.; Chien, S.-C.; Gomes, J. R. B.; Cordeiro, M. N. D. S.; Auerbach, S. M.; Monson, P. A.; Jorge, M. Multiscale Model for the Templated Synthesis of Mesoporous Silica: The Essential Role of Silica Oligomers. Chem. Mater. 2016, 28, 2715-2727.

(23) Berendsen, H.; van der Spoel, D.; van Drunen, R. GROMACS: A message-passing parallel molecular dynamics implementation. Comput. Phys. Commun. 1995, 91, 43-56.

(24) Hess, B.; Kutzner, C.; van der Spoel, D.; Lindahl, E. GROMACS 4: Algorithms for Highly Efficient, Load-Balanced, and Scalable Molecular Simulation. J. Chem. Theory Comput. 2008, 4, 435-447.

(25) Lindahl, E.; Hess, B.; van der Spoel, D. GROMACS 3.0: a package for molecular simulation and trajectory analysis. J. Mol. Model. 2001, 7, 306-317.

(26) van der Spoel, D.; Lindahl, E.; Hess, B.; Groenhof, G.; Mark, A. E.; Berendsen, H. J. C. GROMACS: Fast, flexible, and free. J. Comput. Chem. 2005, 26, 1701-1718.

(27) Hockney, R.; Goel, S.; Eastwood, J. Quiet high-resolution computer models of a plasma. J. Comput. Phys. 1974, 14, 148-158.

(28) Bussi, G.; Donadio, D.; Parrinello, M. Canonical sampling through velocity rescaling. J. Chem. Phys. 2007, 126, 014101.

(29) Berendsen, H. J. C.; Postma, J. P. M.; van Gunsteren, W. F.; DiNola, A.; Haak, J. R. Molecular dynamics with coupling to an external bath. J. Chem. Phys. 1984, 81, 3684-3690.

(30) Martínez, L.; Andrade, R.; Birgin, E. G.; Martínez, J. M. PACKMOL: A package for building initial configurations for 
molecular dynamics simulations. J. Comput. Chem. 2009, 30, 21572164.

(31) Sangwai, A. V.; Sureshkumar, R. Coarse-Grained Molecular Dynamics Simulations of the Sphere to Rod Transition in Surfactant Micelles. Langmuir 2011, 27, 6628-6638.

(32) Yesylevskyy, S. O.; Schäfer, L. V.; Sengupta, D.; Marrink, S. J. Polarizable Water Model for the Coarse-Grained MARTINI Force Field. PLoS Comput. Biol. 2010, 6, e1000810.

(33) Groot, R. D.; Rabone, K. L. Mesoscopic simulation of cell membrane damage, morphology change and rupture by nonionic surfactants. Biophys. J. 2001, 81, 725-736.

(34) Sefcik, J.; McCormick, A. V. Thermochemistry of aqueous silicate solution precursors to ceramics. AIChE J. 1997, 43, 27732784.

(35) Encyclopedia of Surface and Colloid Science, 2nd ed.; Somasundaran, P., Ed.; CRC Press: 2006; Vol. 8; pp 1201-1202.

(36) Brinker, C. J. Porous inorganic materials. Curr. Opin. Solid State Mater. Sci. 1996, 1, 798-805.

(37) Reiss-Husson, F.; Luzzati, V. The Structure of the Micellar Solutions of Some Amphiphilic Compounds in Pure Water as Determined by Absolute Small-Angle X-Ray Scattering Techniques. J. Phys. Chem. 1964, 68, 3504-3511.

(38) Ekwall, P.; Mandell, L.; Solyom, P. The aqueous cetyl trimethylammonium bromide solutions. J. Colloid Interface Sci. 1971, 35, 519-528.

(39) Auvray, X.; Petipas, C.; Anthore, R.; Rico, I.; Lattes, A. X-ray diffraction study of mesophases of cetyltrimethylammonium bromide in water, formamide, and glycerol. J. Phys. Chem. 1989, 93, 74587464.

(40) Tschierske, C. 6 Non-conventional soft matter. Annu. Rep. Prog. Chem., Sect. C: Phys. Chem. 2001, 97, 191-267.

(41) Sugita, Y.; Okamoto, Y. Replica-exchange molecular dynamics method for protein folding. Chem. Phys. Lett. 1999, 314, 141-151.

(42) Firouzi, A.; Atef, F.; Oertli, A. G.; Stucky, G. D.; Chmelka, B. F. Alkaline Lyotropic Silicate-Surfactant Liquid Crystals. J. Am. Chem. Soc. 1997, 119, 3596-3610.

(43) Gomes, J. R. B.; Cordeiro, M. N. D. S.; Jorge, M. Gas-phase molecular structure and energetics of anionic silicates. Geochim. Cosmochim. Acta 2008, 72, 4421-4439.

(44) Galarneau, A.; Renzo, F. D.; Fajula, F.; Mollo, L.; Fubini, B.; Ottaviani, M. Kinetics of Formation of Micelle-Templated Silica Mesophases Monitored by Electron Paramagnetic Resonance. J. Colloid Interface Sci. 1998, 201, 105-117.

(45) Chen, C.-Y.; Burkett, S. L.; Li, H.-X.; Davis, M. E. D. Studies on mesoporous materials II. Synthesis mechanism of MCM-41. Microporous Mater. 1993, 2, 27-34. 